\title{
An index based road feature extraction from LANDSAT-8 OLI images
}

\author{
Sama Lenin Kumar Reddy ${ }^{1}$, C. V. Rao ${ }^{2}$, P. Rajesh Kumar ${ }^{3}$, R. V. G. Anjaneyulu ${ }^{4}$, B. Gopala Krishna ${ }^{5}$ \\ $1,2,4,5$ National Remote Sensing Centre (NRSC), ISRO, Hyderabad, India \\ ${ }^{3}$ Department of ECE, Andhra University College of Engineering, India
}

\begin{tabular}{|c|c|}
\hline Article Info & ABSTRACT \\
\hline Article history: & \multirow{11}{*}{$\begin{array}{l}\text { Road feature extraction from the remote sensing images is an arduous task and has a } \\
\text { significant role in various applications of urban planning, updating the maps, traffic } \\
\text { management, etc. In this paper, a new band combination (B652) to form a road in- } \\
\text { dex (RI) from OLI multispectral bands based on the spectral reflectance of asphalt, is } \\
\text { presented for road feature extraction. The B652 is converted to road index by normal- } \\
\text { ization. The morphological operators (Top-hat or Bottom-hat) uses on RI to enhance } \\
\text { the roads. To sharpen the edges and for better discrimination of features, shock square } \\
\text { filter (SSF), is proposed. Then, an iterative adaptive threshold (IAT) based online } \\
\text { search with variational min-max and markov random fields (MRF) model are used on } \\
\text { the SSF image to segment the roads and non-roads. The roads are extracting by using } \\
\text { the rules based on the connected component analysis. IAT and MRF model segmenta- } \\
\text { tion methods prove the proposed index (RI) able to extract road features productively. } \\
\text { The proposed methodology is a combination of saturation based adaptive thresholding } \\
\text { and morphology (SATM), and saturation based MRF (SMRF), applied to OLI images } \\
\text { of several urban cities of India, producing the satisfactory results. The experimental } \\
\text { results with the quantitative analysis presented in the paper. }\end{array}$} \\
\hline Received Apr 17, 2020 & \\
\hline Revised Jul 16, 2020 & \\
\hline Accepted Sep 25, 2020 & \\
\hline & \\
\hline Keywords: & \\
\hline Band combination of OLI & \\
\hline Road extraction & \\
\hline Saturation & \\
\hline Shock square filter & \\
\hline Top-Hat transform & \\
\hline
\end{tabular}

Corresponding Author:

Sama Lenin Kumar Reddy

National Remote Sensing Centre (NRSC)

Balanagar, Hyderabad, 500 037, India

Email: leninkumar438@gmail.com

\section{INTRODUCTION}

Road feature extraction from remote sensing (RS) images is a challenging and one of the most intensive research topic. Roads are very crucial for transportation, providing many ways of utility for human civilization. The research of road extraction has vital significance for surveying, updating the maps, urban planning, on-line traffic management, geographical information system (GIS), global positioning system (GPS) based road transport and so forth. In the absence of automatic extraction methods, manual road drawing from $\mathrm{RS}$ images requires great effort in terms of cost and time.

RS images provides information for various objects of the earth, based on the spatial and spectral properties. In low resolution (LR) images the roads look like curvilinear structure. From LR imagery, road feature extraction is always a difficult task, mainly in urban places due to the presence of trees, multistory buildings, fly-overs and their shadows are major obstacles irrespective of spatial resolution and sensors. Automatic road extraction from RS images is evolving and most of the approaches are limited in providing solutions with low to medium accuracy. This is due to the factors affecting the imaging conditions like environment (seasonal 
changes), spatial resolution, nature of data [1] and road surface conditions.

Detection and extraction of roads from RS images depend on the width of the road features and spatial resolution of data [2]. Generally, roads constructed by a mix of Asphalt and Gravel or crushed stone, and the spectral reflectance of roads at various conditions (in-situ measurements) has given analysis [3, 4]. New asphalt roads have less reflectance due to the dominance of the hydrocarbon absorption. Aged and paved roads have a high reflectance than new asphalt road due to the erosion of asphalt mix [4].

Using RS images, normalized indices based methods are being used extensively for feature detection like normalized difference water index (NDWI) for water [5], normalized difference vegetation index (NDVI) for vegetation and Built-up area detection by normalized difference building index (NDBI) [6], etc. NDBI index detects both buildings and roads as a built-up area. In the same way, a new band combination is framed to form an index for extract the road features alone from OLI images.

Recently, $[7,8]$ have shared their views on road extraction methods. Most of the methods are using the panchromatic and RGB images. Spectral-spatial classification [9] was implemented to differentiate the road and non-road based on the General adaptive neighborhood mathematical morphology (GANMM) and then morphological profiles [10, 11] created for roads using GANMM. In [12], on segmented objects of very high resolution (VHR) images, object-based frangi's filter (OFF) and object-based shape filter (OSF) are used to enhancing road features and generated training samples to model. In [13], demonstrated that roads from HR images using directional morphological enhancement and segmentation, which depends on the road seed templates homogeneity in different directions. In [14], roads extracted from VHR images by using texture analysis based on the structure feature set standard deviation (SFS-SD) followed by dilation as a preprocessing. Mathematical morphology (MM) used to distinguish the curvilinear features of edges detected by the Canny operator. Linear features enhanced by linearness filtering, which is a combination of Hessian matrix-based filter, local standard variance, and geometrical properties [15]. Supervised classification of SPOT panchromatic images by spatial information and is extracted by morphological openings with various structuring element size according to object size.

After the preprocessing, image segmentation is one of the crucial steps in object-based image analysis (OBIA) and widely used in digital image processing for pattern recognition [16]. A detailed review of RS image classification and feature extraction presented in [17]. The threshold used for binary image segmentation and is calculated based on the histogram, intra-class and interclass variance [18], or optimal value of an image. Segmentation methods are region-based, edge-based and hybrid. Region-based techniques are region growing [19], split-merge [20] which depends on the homogeneity of regions with seed points; and cluster methods (K means, Fuzzy $\mathrm{C}$ means, etc.) depend on the number of classes present in an image. The edge is one of the finest features of an image, and it represents the transition of one object to another object. Many approaches used in literature to detect edge of features by edge operators [21, 22] and edge primitives [23]. In recent literature, researchers proposed new edge extraction methods [24, 25], and these methods are produced better results than classical edge detectors like Canny, Prewitt, etc., but generate the unconnected edges.

A method that segments the images by using optimization is called a hybrid method. This paper adopts two methods, namely the iterative adaptive threshold (IAT) and markov random fields (MRF). IAT, which finds the edges and updates the edges by stochastic gradient descent optimization [26]. MRF is also a stochastic model used for segmentation that uses the neighborhood information [27] and optimized by the iterative condition model (ICM).

The main objective of the work is the extraction of road features (Freeways (Highways), and Arterial (State and District)) from the RS images at the scale of 1:50,000 scale map. OLI images chosen to extract the roads at a 1:50 K scale because at this scale, road widths are varying from 20 meters $(\mathrm{m})$ and above [2]. OLI images have a panchromatic band with $15 \mathrm{~m}$ and multi-spectral bands with $30 \mathrm{~m}$ spatial resolutions. These images cover a swath of $185 \mathrm{~km}$ with a revisit period of 16 days. Its wide swath provides coverage of large areas on the earth with sufficient resolution to distinguish various features like land, farms, forests, urban areas, etc. Here, the OLI pan-sharpened image $(15 \mathrm{~m})$ used in road extraction. In OLI imagery, a large portion of road widths is a minimum of 1,2 , and above 3-pixel roads are very few. For this reason, strategies which can identify wide roads (10-30 pixels) from aerial and HR images [9-15] are not effective. In this paper, the extraction of roads presented using a band combination from multispectral images of OLI based on the spectral range of asphalt to enhance the accuracy of road extraction from LR-RS images.

The rest of the paper describes the proposed methodology in section 2. Various data sets used in this paper presented in section 3 and section 3.1 describes the analysis of the selection of band combinations the 
experimental results with quantitative values shown in section 4.

\section{PROPOSED METHODOLOGY}

The flow diagram of the road extraction proposed methodology using OLI images shown in Figure 1. The thick dotted (Green) outline box represents the saturation based adaptive thresholding and morphology (SATM) method, and thin dotted outline boxes represent the saturation based MRF (SMRF) method. The methodology consists of six stages, and each step described in the following sub-sections.

The proposed methodology is a combination of SATM and SMRF and describes the selection of multispectral band combination for road index given in sections 2.1 and 2.2 In section 2.4, describes the image enhancement by using the SSF filter. The segmentation techniques used in the paper given in 2.5 to segment an image into road and non-road features and road features alone extracted by using the rules based on the shape parameters 2.6.

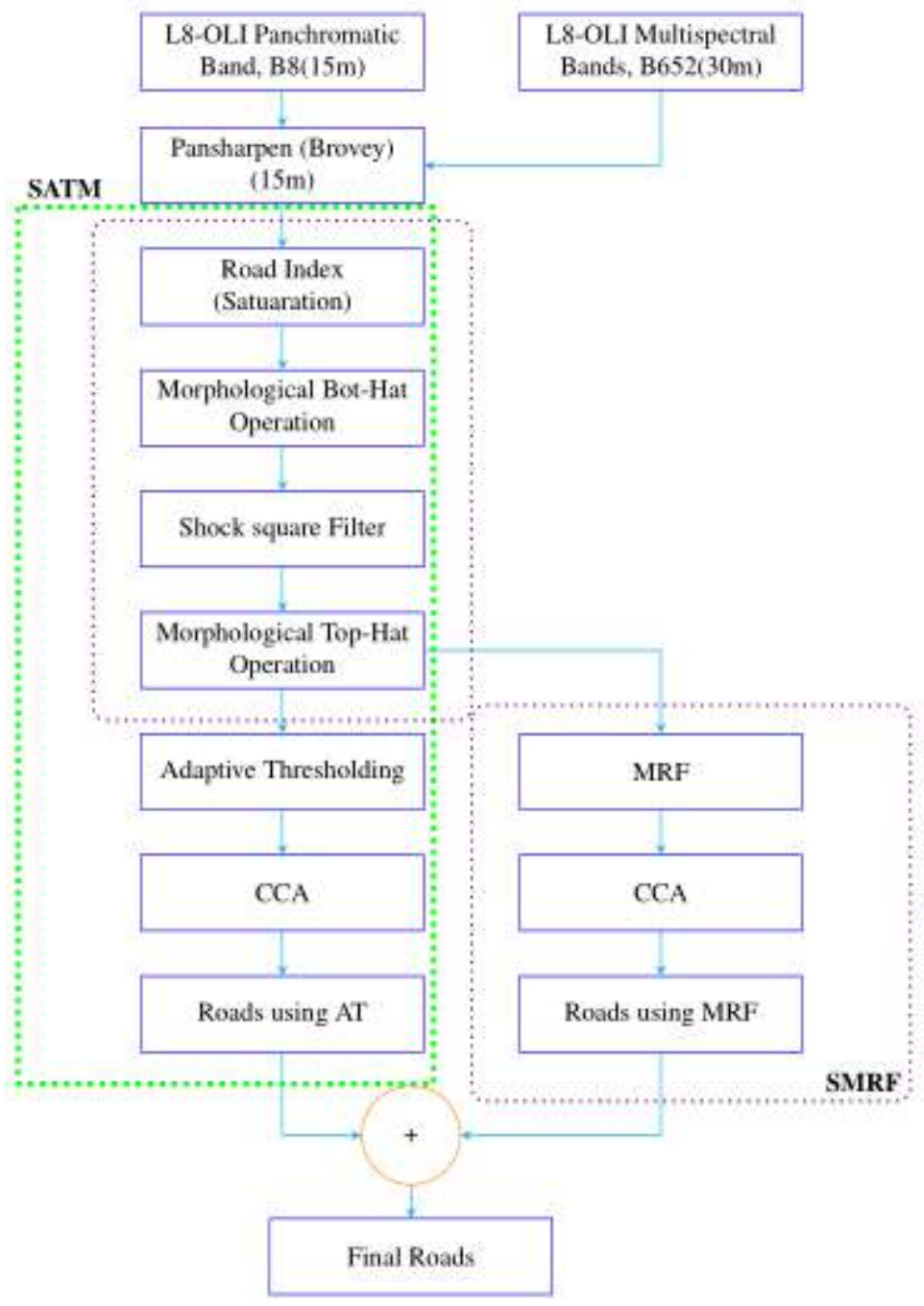

Figure 1. Block diagram of proposed methodology

\subsection{Band selection criteria for road index}

The OLI data is open source and available from USGS earth explorer. The various spectral bands present in the OLI sensor listed in Table 1. 
Table 1. LANDSAT-8 (OLI) sensors and band names

\begin{tabular}{lcr}
\hline Band Name & Wavelength $(\mu m)$ & Spatial Resolution $(\mathbf{m})$ \\
\hline B1 - Coastal aerosol & $0.43-0.45$ & 30 \\
B2 - Blue & $0.45-0.51$ & 30 \\
B3 - Green & $0.53-0.59$ & 30 \\
B4 - Red & $0.64-0.67$ & 30 \\
B5 - NIR & $0.85-0.88$ & 30 \\
B6 - SWIR 1 & $1.57-1.65$ & 30 \\
B7 - SWIR 2 & $2.11-2.29$ & 30 \\
B8 - Panchromatic & $0.50-0.68$ & 15 \\
\hline
\end{tabular}

Compared to the usage of the panchromatic image for feature extraction, the multispectral image contains more information in several spectral bands which used for advantage. Each band in a multispectral image corresponds to a band of wavelengths in visible, IR, and near infra-red (NIR) regions. From the available multi-spectral bands of OLI, we propose a band combination to form a road index (RI) for road feature extraction.

The reflectance of asphalt is more in NIR, short wave infra-red (SWIR) in [4]. USGS Spectroscopy Lab provided, the spectral reflectance of various minerals, liquids, vegetation, etc. Asphalt road spectral values are available from https://speclab.cr.usgs.gov/. Figure 2 shows the spectral variation from $0.35 \mu \mathrm{m}$ to $2.5 \mu \mathrm{m}$. Spectral reflectance of asphalt feature and other features from the OLI image shown in Figure 3. From this, we can observe the asphalt reflectance variation is almost matching with the provided spectra as shown in Figure 2 in the bands NIR and SWIR.

In visible band region $(0.40 \mu \mathrm{m}$ to $0.70 \mu \mathrm{m})$ asphalt has low reflectance compared to NIR and SWIR regions $(0.85 \mu \mathrm{m}$ to $2.5 \mu \mathrm{m})$. In the spectral range of $1.8 \mu \mathrm{m}$ to $2.5 \mu \mathrm{m}$, which is above the SWIR wavelength, the reflectance of the asphalt roads is varying. From this, it concludes that asphalt roads have a favorable spectral response in NIR and SWIR wavelengths.

The OLI sensor has different bands of wavelengths given in Table 1. According to Spectral Reflectance of asphalt road from Figures 2 and 3, the suitable bands for road detection in OLI are NIR, SWIR1, and SWIR2 bands.The NIR $(0.85 \mu m-0.88 \mu m)$ and SWIR1 $(1.57 \mu m-1.65 \mu m)$ present in OLI have narrow spectral bandwidth with good reflectance for road features (linearly increasing). In SWIR2 $(2.11 \mu \mathrm{m}-2.29 \mu \mathrm{m})$ from $2.11 \mu \mathrm{m}$ to $2.2 \mu \mathrm{m}$ reflectance of the roads is increasing and in the range $2.2 \mu \mathrm{m}$ to $2.3 \mu \mathrm{m}$ it is decreasing result in uncertainty in feature recognition. Additionally, the SWIR band can also distinguish the moisture content of soil and vegetation. The blue and green bands are having very low reflectance of asphalt road in the entire wavelength range.

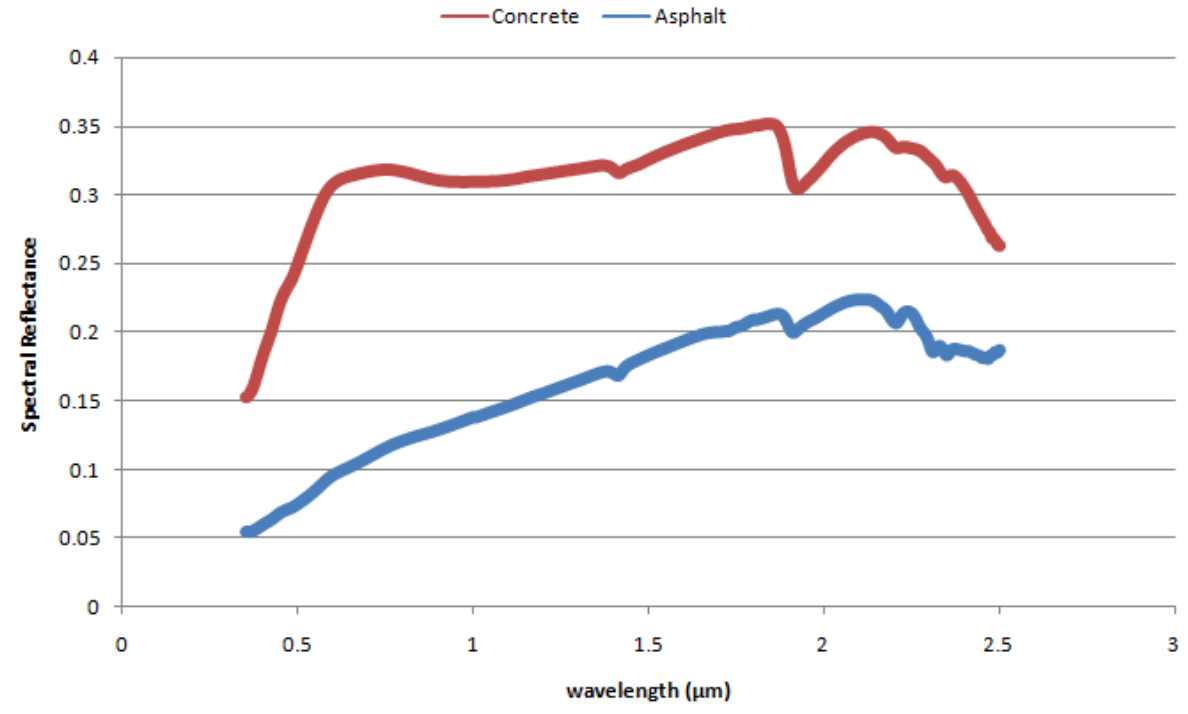

Figure 2. Spectral reflectance vs. wavelength of asphalt and concrete 


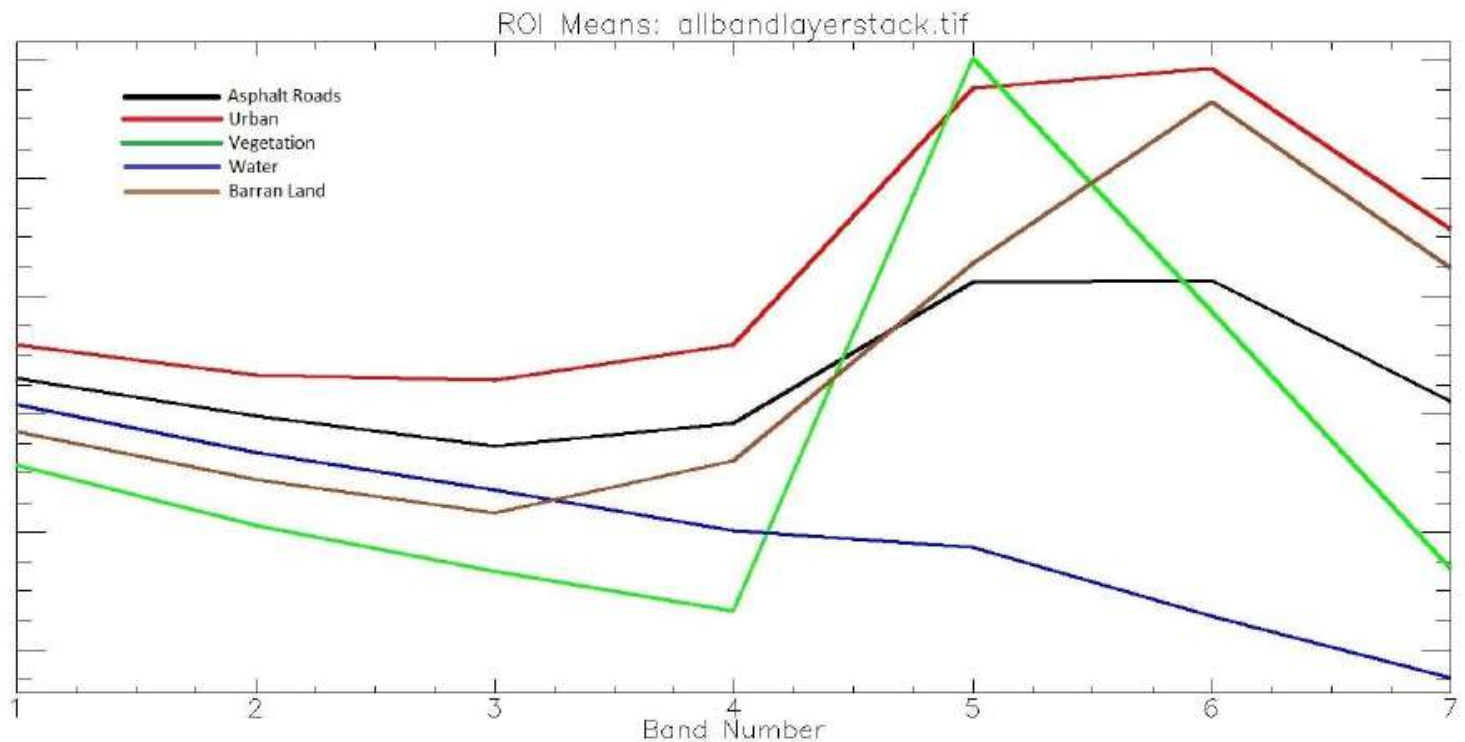

Figure 3. Spectral reflectance vs. bands in OLI

From the available OLI multispectral bands, the bands BLUE, NIR, SWIR1 used to form an RGB image. These bands proved to be the better combination for road extraction in the section 3.1. The bands B6, B5 and B2 are stacked, as RGB (B652) image. A fusion of B652 image with the Panchromatic band (B8) carried out using Brovey transform. This method [28] is chosen as it is faster and does not affect the band spectral relationship for road features as shown in Figure 4 with very fine spatial details.

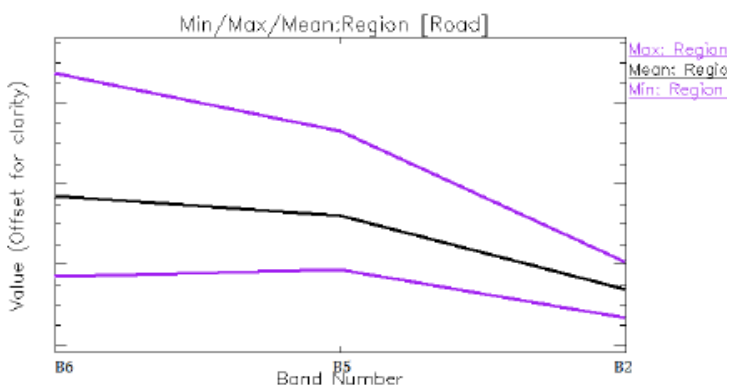

(a)

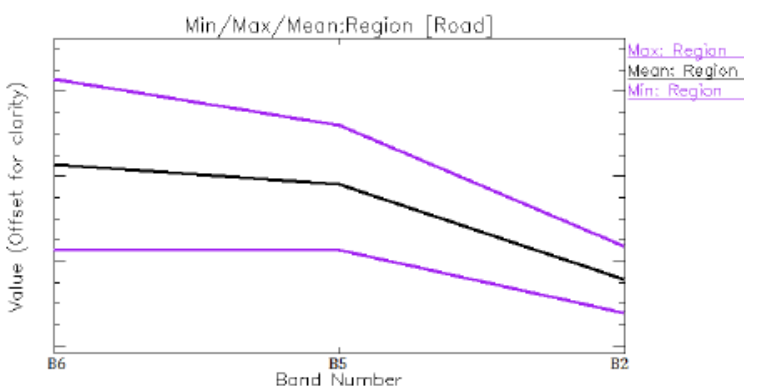

(b)

Figure 4. Spectral response of Asphalt from OLI, (a) B652 image (b) Fused image

\subsection{Road index: Saturation}

HSI color model most widely used in digital color image processing and developed by [29]. The road index (RI) component derived as saturation (S) formulae from the selected band combination B652, and the roads represented in uniform and with low values compared to surrounding features due to minimum reflectance value from three bands is dividing with the summation of all band values. In the RI (or S) image, roads show high discrimination from the other features. $\mathrm{S}$ image has normalized values within the range of [0,1]. Here, $\mathrm{S}$ is used (as termed in the paper) as road index (RI) and defined as the following 1 for B652.

$$
S=R I=1-\frac{3 * \min (S W I R 1, N I R, B L U E)}{(S W I R 1+N I R+B L U E)}
$$




\subsection{Morphological transforms}

The MM is a set theory approach consisting of union, intersection and complement. Top-hat (TH) transform used to enhance the objects smaller than the structuring element. It preserves sharp peaks and improves contrast. There exist two types of TH transform. White TH or TH transform, which highlights the 'Bright' features and defined as subtraction of morphological opening (o) image from the initial (before opening) image. Black TH or Bottom TH (simply bot-hat (BH)) transform defined as dual as the difference between morphological closing $(\bullet)$ image and the input image. It highlights the 'Dark' features that are smaller than the structuring element.

$$
\begin{aligned}
& T H=S_{t h}=S-(S \circ B) \\
& B H=S_{b h}=(S \bullet B)-S
\end{aligned}
$$

Where, $S_{t h}$ represents the TH applied on RI image , B is structuring element 'disk' with (suitable) radius, and $S_{b h}$ represents BH. TH and BH transforms are uses in various image processing steps in pre or post-processing of the feature extraction process. BH transformation applied to the RI image, which highlights the roads and suppresses the remaining features.

\subsection{Shock square filter}

Shock filter is one of the classes of morphological enhancement method and given by [30] as

$$
I_{t}=-\operatorname{sgn}(\triangle I)|\nabla I|
$$

Here, I represent any gray-scale image. The Laplacian of I i.e., $(\triangle I)$ is act as edge detector. The $\triangle I$ replaced by $\triangle(G \circledast I)$ in [31]. In this notation $\mathrm{G}$ is Gaussian and $\circledast$ is convolution.

$$
I_{G}=-\operatorname{sgn}\left(\triangle\left(G \circledast S_{b h}\right)\right)\left|\nabla S_{b h}\right|
$$

Here, $S_{b h}$ is $\mathrm{BH}$ of $\mathrm{S}$ image as derived in (3).

The shock filter used to sharpen edges but forms maze-like structures in regions [32]. To eliminate this, we proposed a filter, which is a combination of Shock filter [31, 30] followed by a modified shock filter. This smooth the regions as well as keep the sharp edges of features. feature).

Here, the motive is to sharpen the edges of the image and to smooth the regions (inside the objective

The direction of shocks measured by using the hyperbolic tangent function $T_{s h}$ described in [32] on the $\mathcal{E}$, which is edge detector. The modified shock filter $I_{s h}$ is

$$
\begin{gathered}
I_{s h}=\left(1-S \cdot \frac{\operatorname{sgn}(\mathcal{E})}{2}\right)\left|\nabla I_{G}\right| \\
\operatorname{sgn}(x)= \begin{cases}1, & \text { if } x>0, \\
0, & \text { if } x=0, \\
-1, & \text { if } x<0 .\end{cases}
\end{gathered}
$$

where $(\triangle I)$ replaced by Weickert's coherence shock filter [33]. It is one of the best edge detectors and multiplied by the Road index (S). In this way, the regions smoothed along with sharp edges in the image.

$$
T_{s h}(\mathcal{E})=\frac{\left(1+\tanh \left(\lambda\left(\left(\frac{1-\operatorname{sign}(\mathcal{E})}{2}\right)-0.5\right)\right)\right)}{2}
$$

where $\lambda$ designed for tuning the sharpness of curve function; hence, it set as the value 6 (fixed). $\mathcal{E}$ is an edge detector, as described in Weickert's coherence shock filter [33]. Let $I=S_{b h}$, then

$$
\mathcal{E}=\left(I_{x}^{2} I_{x x}+2 I_{x} I_{y} I_{x y}+I_{y}^{2} I_{y y}\right)
$$


The edge detection is performed on the shock filtered image by using (5). An-isotropic Shock filter [32] is represented as

$$
I_{s h}^{\prime}=T_{s h} \cdot D\left(I_{b h}\right)+\left(1-T_{s h}\right) \cdot E\left(I_{b h}\right)
$$

$D\left(I_{b h}\right)$ and $E\left(I_{b h}\right)$ are dilation and erosion of BH image. SSF is able to get sharp edges and enhance the feature objects. Also, it is useful in removing the connected noise to the feature. Without SSF, the extracted roads are having connected noise like building and spike ridges. By using SSF, it is producing better discrimination as shown in Figures 5a and 5b, i.e., roads in the image are enhanced and sharpen edges (highlighted in red color boxes). To suppress the other elevated features by the SSF, the TH transform is applied on the SSF image as aforementioned in section 2.3.

$$
I_{T H}^{\prime}=I_{s h}^{\prime}-\left(I_{s h}^{\prime} \circ B\right)
$$

where $I_{s h}^{\prime}$ is SSF image and B is structuring element 'disk' with suitable radius.

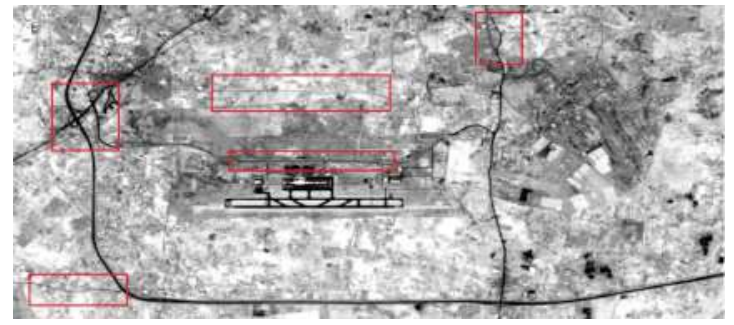

(a)

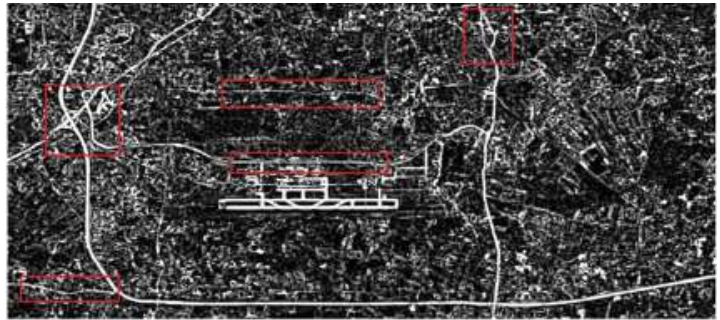

(b)

Figure 5. (a) Road Index i.e., Normalization of B652, (b) SSF on Bot-Hat of S (RI) image

\subsection{Segmentation methods}

An IAT algorithm is an edge-based image thresholding method and edges updated by iterative using the stochastic gradient descent optimization method based on the variational min-max principle [26] performed on the $I_{T H}^{\prime}$ image to get the segmented binary image. It helps in the extraction of roads accurately. This process termed as SATM.

A model, MRF [27] is also used for the segmentation of road features on $I_{T H}^{\prime}$ image, when there is no prior information about the model parameters. MRF depends on the neighborhood information for its probability distribution. The parameters estimated by using the expectation-maximization (EM) algorithm. The segmenting classes are known, and the label image generated by using the optimal threshold on TH, i.e., TH of SSF image. This process termed SMRF. MRF model provides the solution with maximum a posterior (MAP) [34] estimation by the maximizing label class and minimizing using the posterior energy function. The parameters (mean and variation) estimated by using the EM algorithm [35].

\subsection{Connected component analysis}

In the final step, by using the connected component analysis (CCA) [36], the segmented binary image is labeled. For each label, the shape parameters like extent (Ex), eccentricity (Ec) computed [37]. The extent defined as a ratio number of pixels in the label to the bounding box of the same label. The label whose Ex value is $\leq 0.12$ and also Ec value is $\geq 0.99$ considered as road label for extraction of elongated roads. The road features in OLI images look like curvilinear, elliptical, etc., for this reason, for each label, the shape features like shape index (SI) and density (D) computed. The SI with high values at the same time the D has moderate values of the label taken as road features from the labeled image.

\section{DATA SETS}

Data sets of OLI covering various urban areas used to extract the roads, which are level-1 precision and terrain (L1TP) corrected used for road extraction. In this study, four OLI data sets covering three major 
urban areas of Hyderabad, Chennai, Bangalore, and one data set covering the rural areas of Rajasthan used to evaluate the performance of the proposed methodology. The used scene id along with their date of pass for each chosen area given in Table 2. All images mentioned in the paper are top of atmosphere (TOA) corrected. The size of the image represents the subset scene image.

Table 2. Data sets with scene ID's and date of pass

\begin{tabular}{lcrrrr}
\hline S.No. & Date of Pass & sub-scene & Scene ID & Size of Image \\
\hline 1 & $2016-04-05$ & Hyderabad & LC81440482016096LGN00 & $3291 \times 3099$ \\
2 & $2017-02-21$ & Chennai & LC81420512017052LGN00 & $1586 \times 1994$ \\
3 & $2017-02-03$ & Bangalore & LC81440512017034LGN00 & $1978 \times 1888$ \\
4 & $2018-05-09$ & Rajasthan & LC81480402018129LGN00 & $600 \times 500$ \\
\hline
\end{tabular}

\subsection{Analysis on various band combinations}

As given in section 2.1 for road feature extraction, the chosen band combination is B652. In this section, the various band combinations of OLI experimented with the proposed methodology. A few interest band combinations are B652, B653, B654, B752, and B762 selected for analysis and shown in Figure 6 for importance in road extraction. The band combination B432 is a natural color image, used in this methodology. B432 combination was not able to produce even major roads as well. By using the proposed methodology, B432 is not useful for road detection from OLI.

The band combinations B542 and B654 are false color composite (FCC) images. In the B4 band, the concrete has high, and asphalt has low reflectance with each other, due to which converted $\mathrm{S}$ image has mixed features (building and roads). The B542 and B654 are helpful in the extraction of major roads only but not able to recognize other roads. The B654 combination image was shown in Figure 6i and corresponding results are RI index image, Optimal Threshold from $I_{T H}^{\prime}$ image and SATM result shown in Figures $6 \mathrm{j}$, 6k and 61 respectively.

The B752 band combination, not able to produce urban area roads of a few major roads also missing. Due to B7 has uncertainty in the reflectance of concrete and asphalt. From Figure 3, B7 has a low reflectance of asphalt and almost equal to band B2. In conversion to S, most of the road values mixed with the barren and built-up areas, the segmentation methods are not able to separate the road features. The corresponding results of B752 are shown in Figures 6m, 6n, 6o and 6p.

The band combination B762 is able to detect the major roads, and few in urban area roads using the proposed method. Because of the asphalt and concrete reflectance are higher in bands B7, B6 and low in B2. The S of B762 is also useful in road detection, but only a few roads are missing. These missed roads detected by B652. Also, the B653 band combination able to extract roads, but B652 detects more roads with good accuracy and smoothness. This can be observed in Figure $6 \mathrm{t}, 6 \mathrm{~h}$ and $6 \mathrm{~d}$. Based on the experimental results and analysis, the chosen band combination is B652 for road extraction.

One of the widely used methods for the selection band combination is the optimal index factor (OIF), which depicts the correlation of bands that have the highest to lowest OIF band combinations. By using this, it observed that the band combined with both SWIR1 and NIR bands have the highest OIF values. The spectral correlation plot between NIR and SWIR bands with the corresponding optical image shown in Figure 7 by using ENVI. From this, the water bodies have the highest correlation as well as vegetation. Also, it observed that asphalt has a high correlation from the other features, especially in urban areas. From this, both the bands are most useful to form the RI index.

According to OIF, the B651, B652, and B654 band combinations have the highest OIF values from all combinations. In the two (B651 and B652) combination, the B2 band is a regular band in all types of sensors, and B1 is a rarely using sensor in RS. Hence, the chosen band combination is B652. The various band combinations of original images with the RI index and corresponding results of optimal threshold and SATM shown in Figure 6. 


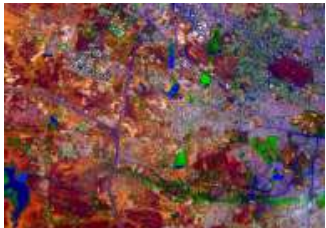

(a)

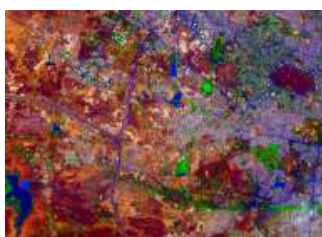

(e)

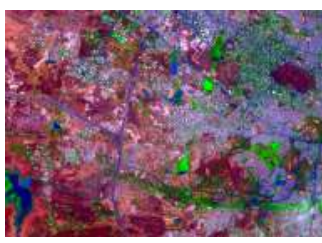

(i)

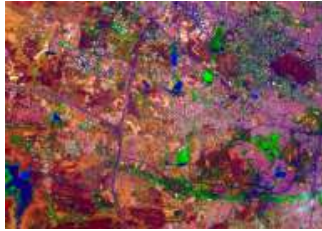

(m)

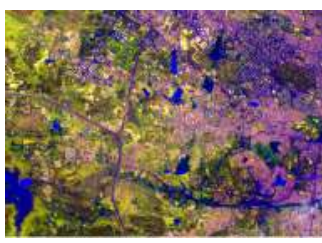

(q)

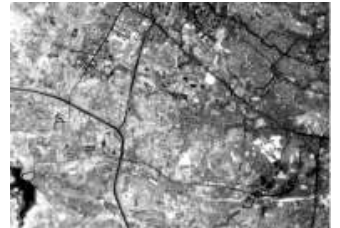

(b)

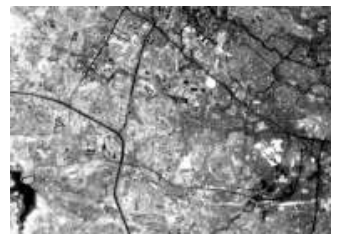

(f)

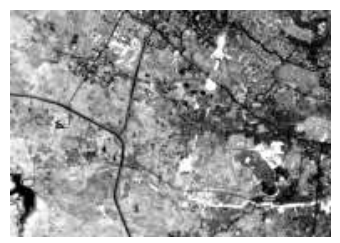

(j)

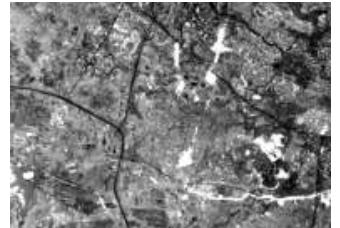

(n)

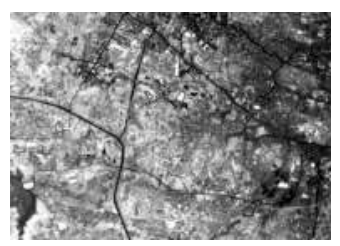

(r)

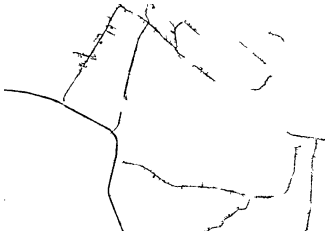

(c)

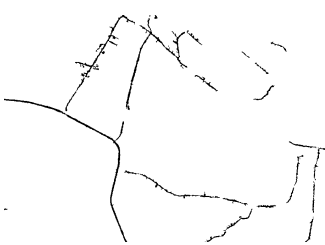

(g)

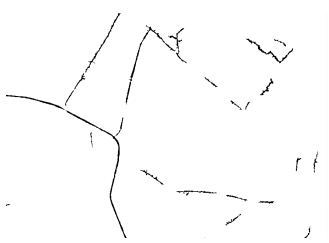

(k)

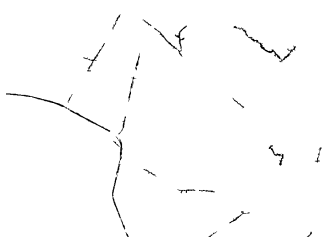

(o)

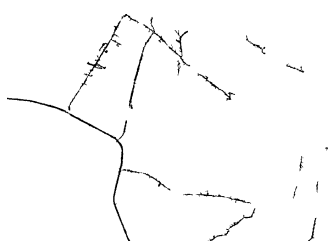

(s)

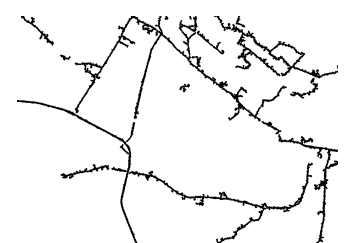

(d)

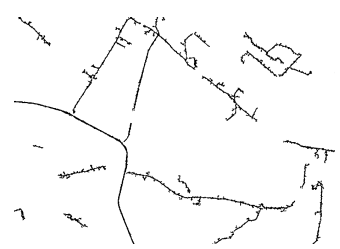

(h)

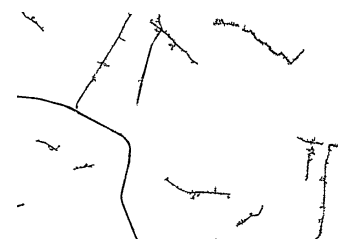

(1)

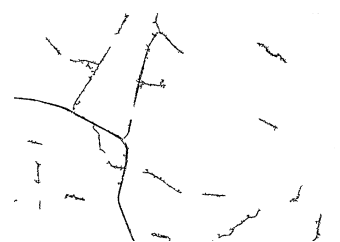

(p)

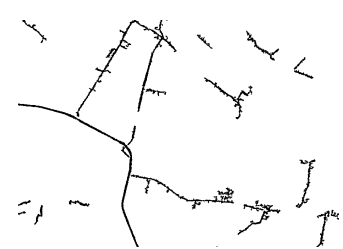

(t)

Figure 6. (i). OLI data sets of band combinations (a) 652 (e) 653 (i) 654 (m) 752 (q) 762 , (ii). RI of (i), (iii). Using Optimal Threshold on RI of BH images (i) (iv) SATM

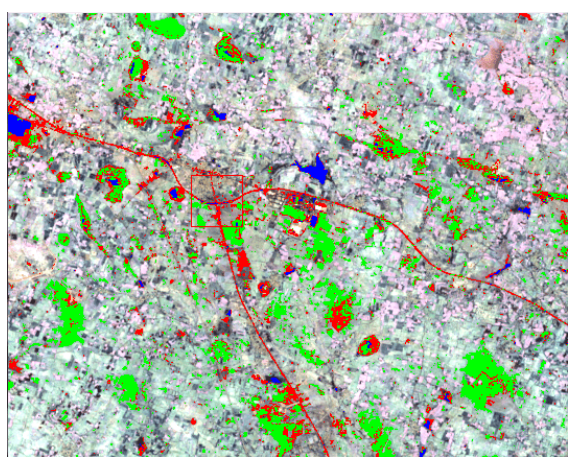

(a)

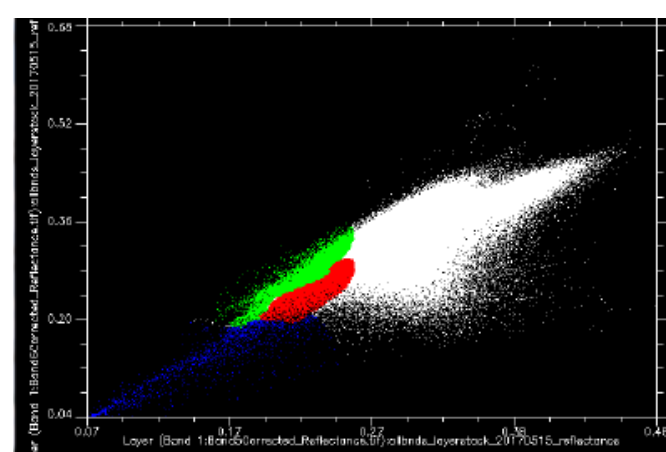

(b)

Figure 7. (a) OLI B652 image (b) Spectral plot of B6 Vs. B5 


\section{EXPERIMENTAL RESULTS AND DISCUSSION}

The proposed methodology is applied to sub-scene images of a full scenes, as mentioned in Table 2. All these sub-scene images are multispectral band combination of B652, as proposed in section 2.1 and based on analysis in section 3.1. The small scenes of sub-scenes Figure 8a (Chennai) and Figure 9a (Hyderabad) chosen for proposed methodology verification, has the roads and as well as water bodies. The proposed methodology consists of two methods are SATM and SMRF. One method SMRF applied to Figure 8a, for extraction of roads, but observed that water body edges also extracted. SATM applied to Figure 9a, also observed that water body edges are present along with roads. A method proposed for the water body and water canal extraction [38] used to remove the water body edges from the images.

\subsection{Removal of water body edges}

As mentioned above, by using the proposed methodology, the water body edges are also extracted as roads. Due to the water body (lakes, reservoirs and canals) boundaries are man-made structures, by concrete, gravel, and stone. These water body edges removed by the proposed method MNDWI2 of water bodies in the same images and dilated. That is, MNDWI2 [38] index used to extract the water bodies and water canals from the same image. From this method, extracted water bodies from the images shown in Figures $8 \mathrm{c}$ and $9 \mathrm{c}$ (SR is $30 \mathrm{~m}$ ). After extraction of these features, image interpolated by cubic convolution to the SR of $15 \mathrm{~m}$ and dilated by the structure element 'disk' with radius 2 to match with edges. Wherever water body edges present (if water body positions are matches in both images $8 \mathrm{~b}$ and $8 \mathrm{c}$ ) are removed by using logical operators. Water body edges removed image shown in Figure 8d and similar procedure applied for Figure 9. Further results shown in subsection 4.2 used the same methodology for the removal of water bodies edges from the resultant images of the proposed methodology.

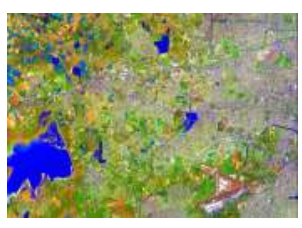

(a)

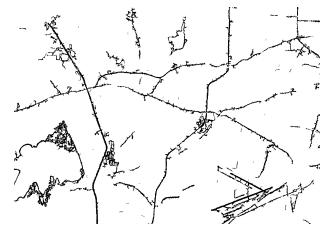

(b)

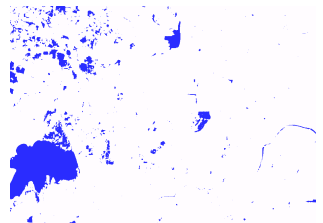

(c)

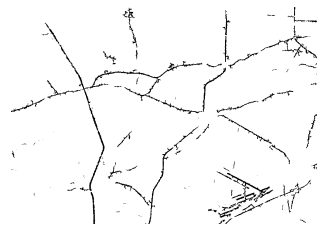

(d)

Figure 8. (a) OLI B652 image (Chennai) (b) SMRF (c) MNDWI2 [38] (d) Final roads

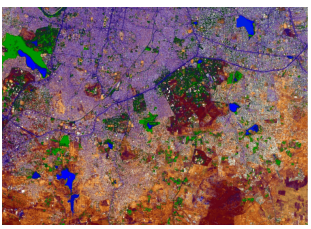

(a)

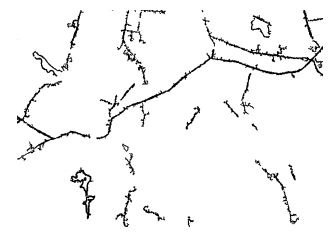

(b)

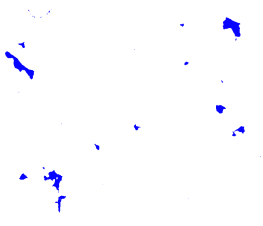

(c)

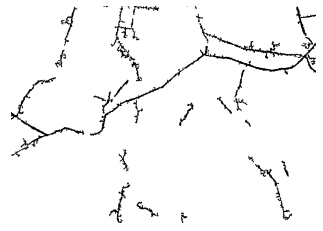

(d)

Figure 9. (a) OLI B652 image (b) SATM (c) MNDWI2 [38] (d) Final roads

\subsection{Results and discussion}

The proposed methodology performed on OLI data sets given in Table 2. The sub-scenes of data sets taken as which covers the urban areas and one sub-scene (Rajasthan) with arid type rural area. The different steps involved in the proposed methodology resultant image shown in figures.

As mentioned in section 2.1 the proposed new multi-spectral band combination B652 sub-scene images of full scenes as shown in Table 2 are shown in Figures 10a, 11a and 12a. All the mages mentioned in the paper are pan-sharpened by the Brovey method with SR of $15 \mathrm{~m}$. These images are converted into indexed RI image by using the 1 and are shown in Figures $10 \mathrm{~b}, 11 \mathrm{~b}$ and $12 \mathrm{~b}$. These results illustrate the proposed index (RI) image, and the roads having low values at the same time differentiating from the other features. To enhance the road feature, BH is applied and elevates road features as well as suppress other features. 
Figures 10c, 11c, and 12c, illustrates the proposed filter (After applying the BH and SSF), sharpen the road edges as well as smooth the road regions.

SSF images segmented by IAT, Otsu, and MRF, which segment as the road and non-road feature. From these segmented images, road features alone extracted by using CCA and are shown in Figures 10d, 10e, and 10f. As described in section 4.1 the water body's edges also extracted as roads due to the features are like roads and those water bodies removed from segmented images.

Figure $10 \mathrm{~g}$ describes the proposed methodology of the combined results of SATM and SMRF. In combining the results, we can observe that the roads little more than individuals. Also observed, roads which are freeways and arterial roads extracted with high accuracy. Similarly, road features are extracted from the remaining images and given in Figures 11, 12 and 13. SATM results are shown in Figures 11d and 12d. Figures 11e and 12e represents the SMRF results. The final results (Combined images) of proposed methodology are shown in Figures $11 \mathrm{f}$ and $12 \mathrm{f}$.

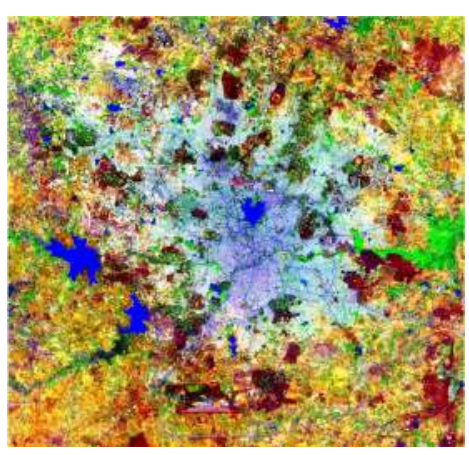

(a)

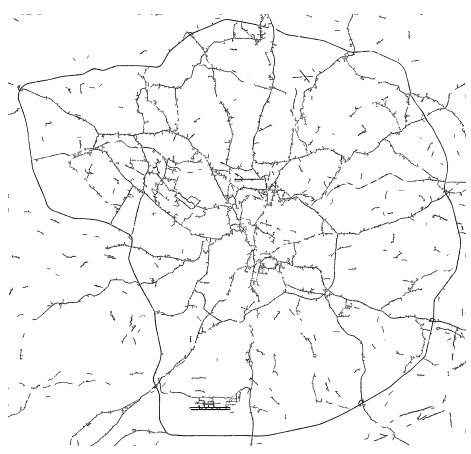

(d)

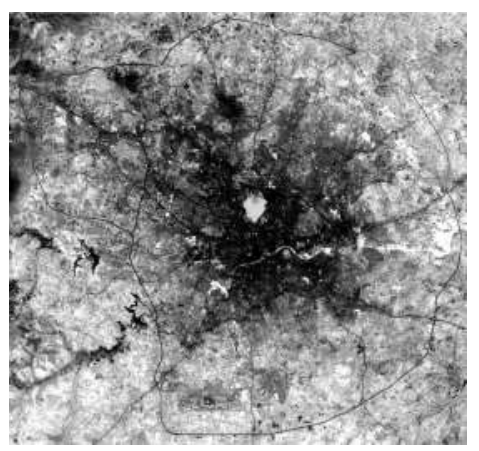

(b)

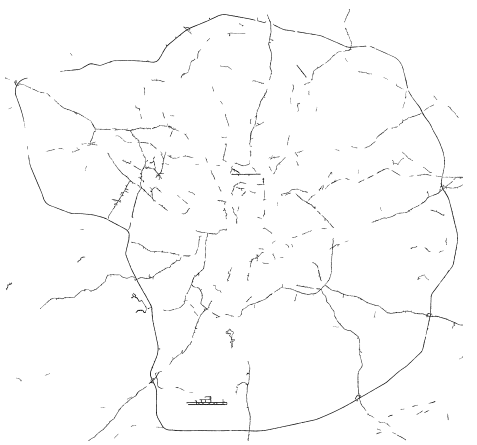

(e)

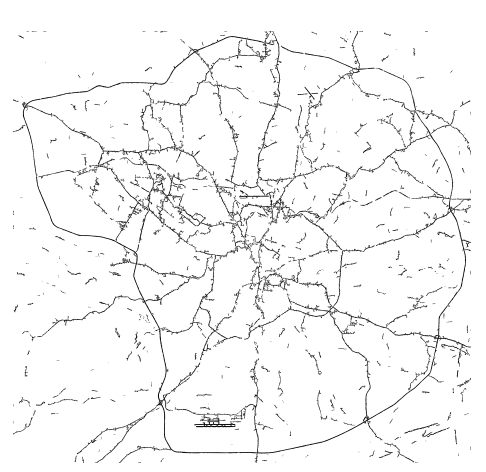

(g)

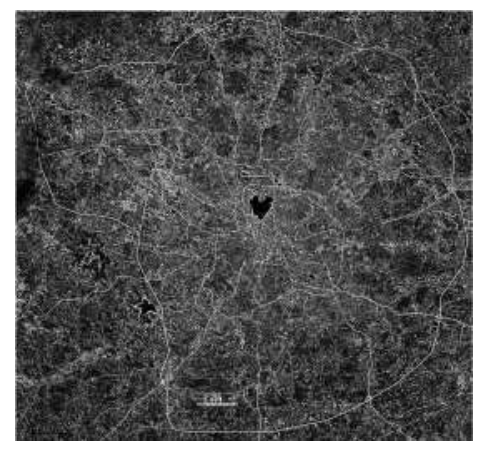

(c)

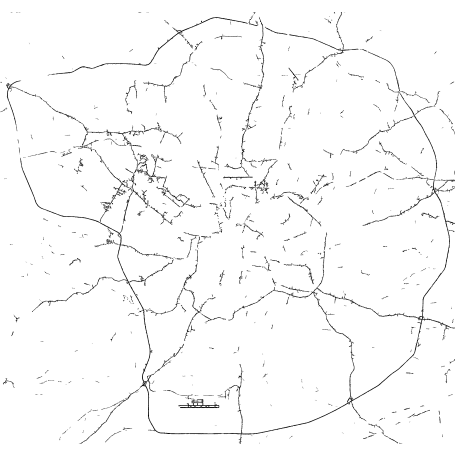

(f)

Figure 10. (a) OLI B652 (Hyderabad) image, (b) Road index, (c) SSF, (d) SATM, (e) Optimal threshold, (f) SMRF, (g) Proposed methodology 
In OLI, pan-sharpened images, each pixel represents $15 \mathrm{~m}$ on the ground. Generally, road widths are $7 \mathrm{~m}$ (two-lane), $15 \mathrm{~m}$ (4 lanes), $30 \mathrm{~m}, 45 \mathrm{~m}$ and vary. Highway roads have 4 to 6 (or more) lanes with a varying width from $15 \mathrm{~m}$ to $45 \mathrm{~m}$, which have smooth curves represents one to 3 pixels in the image. District (Arterial) roads are mostly 2 or 4 lanes with paved or unpaved roads varying width from $8 \mathrm{~m}$ to $20 \mathrm{~m}$. Minor (residential) roads are mostly irregular with less than $7 \mathrm{~m}$ width.

By using the proposed methodology results illustrates in the Figures 10, 11 and 12, and the roads width $>22 \mathrm{~m}$ (1.5 pixels) are extracted consistently. The roads with $7 \mathrm{~m}(1 / 2$ pixel) width are also extracted based on contrast and separation of road and background. These images show the proposed methodology extracting the roads with good accuracy.

Figure 13a represents a sub-scene of the rural area (residential type) roads of Rajasthan with a band combination of B652. RI and enhanced images are shown in Figures 13b and 13c. In the arid type of conditions, roads highlighted due to high asphalt reflectance and observed the width of the roads is varied from $7 \mathrm{~m}$ to 3 $\mathrm{m}$ shown in Figure 13. These types of roads also are extracted efficiently by the proposed method, as shown in Figure 13d. The road widths less than $7 \mathrm{~m}$ also extracted in such arid areas due to obstacles not present beside roads.

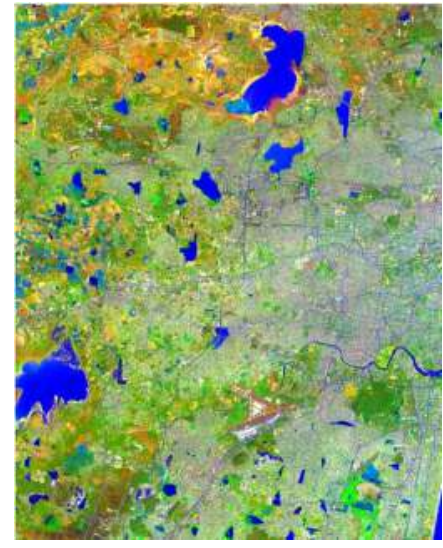

(a)

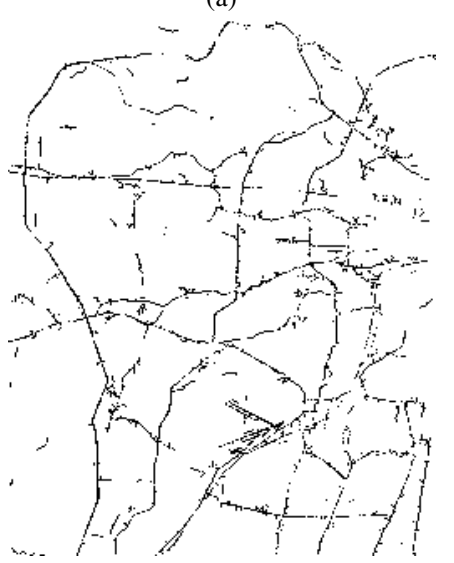

(d)

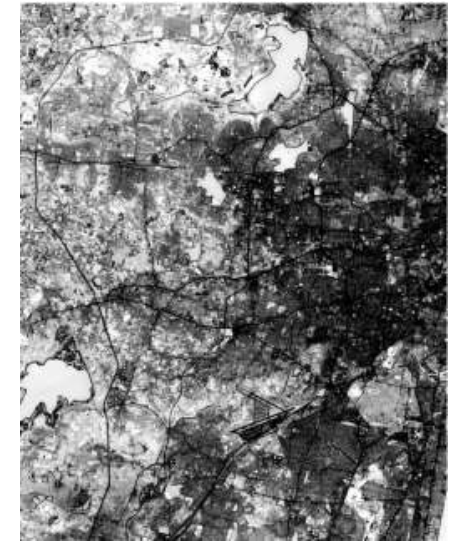

(b)

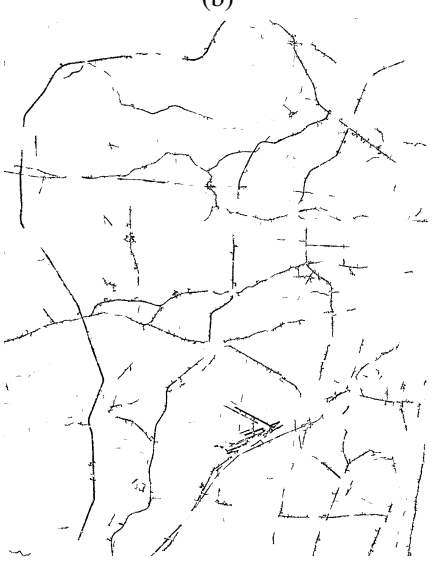

(e)

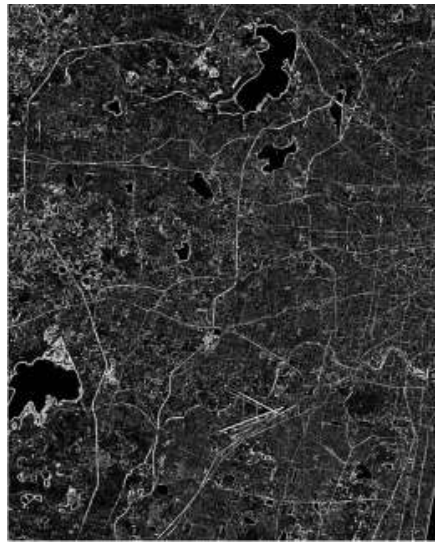

(c)

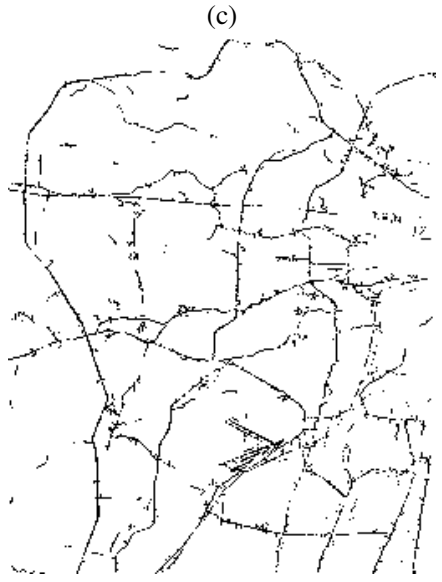

(f)

Figure 11. (a) OLI B652 (Chennai) image, (b) Road index, (c) SSF, (d) SATM, (e) SMRF,

(f) Proposed methodology

From the results, it observed that more than $15 \mathrm{~m}$ width of roads extracted with 100 percent, when not present multi-storey buildings and trees beside the road. The roads width less than $30 \mathrm{~m}$, which are present in between the buildings and trees, pixel reflectance changes for roads due to having the high reflectance of buildings and trees, and not able to identify as the road in such dense urban areas.

The observed gaps in between the roads because of not enough width of roads, not consistent and urban area roads with less than $30 \mathrm{~m}$. Gaps with less than 3 pixels in between roads connected, by opening 
and closing operators of morphology used. Gaps less than 10 pixels in between roads connected by the circle method, that is, at every endpoint placed a circle ( with diameter 10) and thinned by morphological Operation. Where two endpoints of circles are connected, there itself connect, and remaining are left. The proposed method with gaps filled results converted into vector form by using ArcGIS 10.2, and these results used for validation.

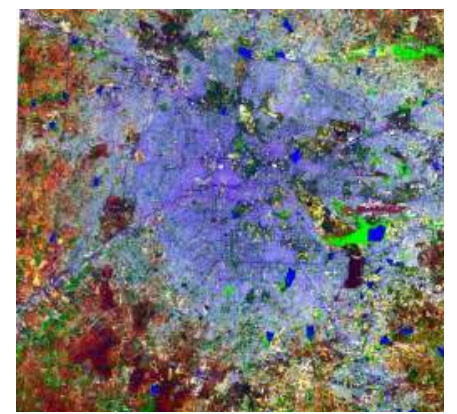

(a)

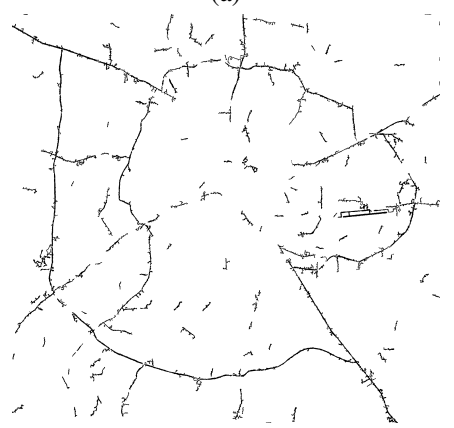

(d)

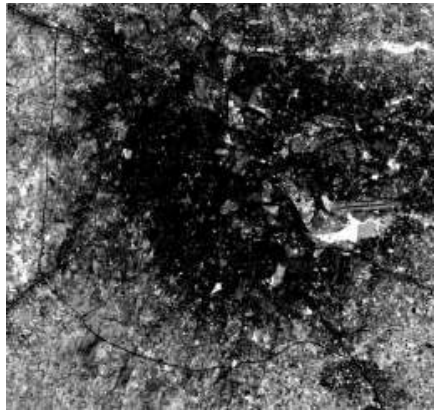

(b)

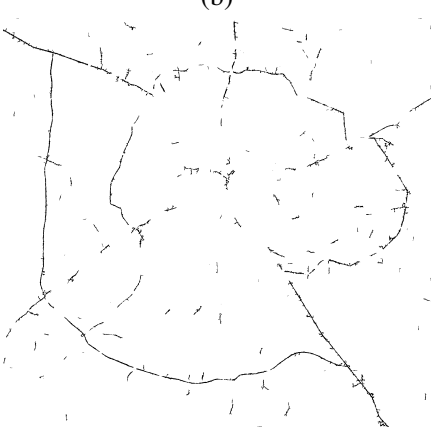

(e)

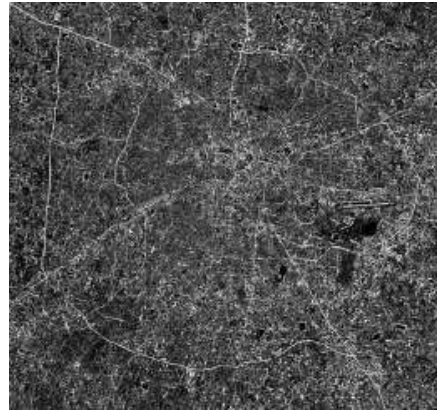

(c)

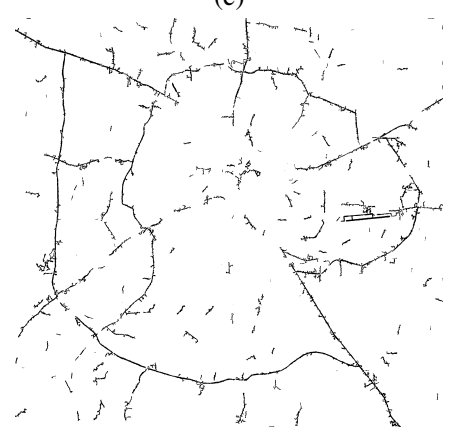

(f)

Figure 12. (a) OLI B652 (Bangalore) image , (b) Road index, (c) SSF, (d) SATM, (e) SMRF, (f) Proposed methodology

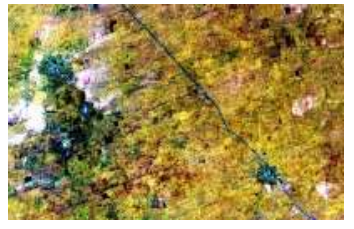

(a)

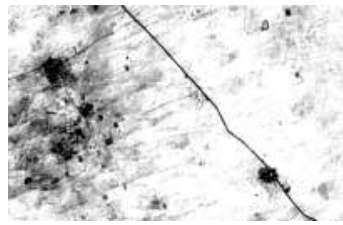

(b)

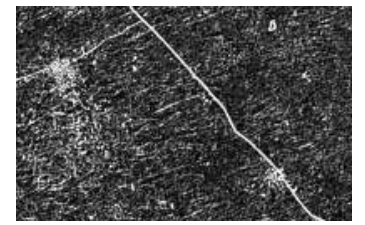

(c)

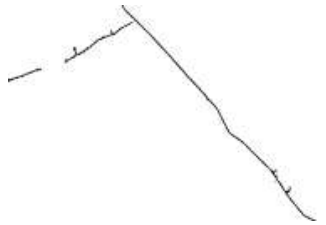

(d)

Figure 13. (a) OLI B652 (Rajasthan)image (b) Road index (c) SSF (d) Proposed methodology

The accuracy of the proposed methodology in terms of length of extracted roads using proposed one of method SATM and Reference lengths(manually drawn on OLI test case images with QGIS) given in Table 3. The accuracy of SATM method for sub-scenes given in Table 4. Also, the length of extracted roads by the proposed methodology (SATM + SMRF) in Table 5 and accuracy presented in in Table 6 for the same. From these tables, the index based methods SATM and proposed methodology able to extract the road features with an overall accuracy of $85 \%$.

Table 3. Accuracy in Length (in km) of Roads for SATM

\begin{tabular}{cccc}
\hline sub-scene & Hyderabad & Chennai & Bangalore \\
\hline SATM & 1117 & 436 & 304 \\
Reference & 925 & 411 & 322 \\
TP & 869 & 390 & 249 \\
FN & 56 & 21 & 73 \\
FP & 248 & 46 & 55 \\
\hline
\end{tabular}


Table 4. Accuracy of SATM

\begin{tabular}{cccc}
\hline sub-scene & Precision (\%) & Recall (\%) & F1-Score \\
\hline Hyderabad & 77.80 & 93.95 & 85.11 \\
Chennai & 89.45 & 94.89 & 92.09 \\
Bangalore & 81.91 & 77.33 & 79.55 \\
\hline
\end{tabular}

Table 5. Length (in $\mathrm{km}$ ) of extracted roads by proposed methodology

\begin{tabular}{cccc}
\hline sub-scene & Hyderabad & Chennai & Bangalore \\
\hline SATM+SMRF & 1209 & 463 & 325 \\
Reference & 925 & 411 & 322 \\
TP & 910 & 399 & 263 \\
FN & 15 & 12 & 59 \\
FP & 299 & 64 & 62 \\
\hline
\end{tabular}

Table 6. Accuracy of proposed methodology

\begin{tabular}{cccc}
\hline sub-scene & Precision $(\%)$ & Recall $(\%)$ & F1-Score \\
\hline Hyderabad & 75.27 & 98.38 & 85.29 \\
Chennai & 86.18 & 97.08 & 91.30 \\
Bangalore & 80.92 & 81.68 & 81.30 \\
\hline
\end{tabular}

\subsection{Validation}

Reference roads are obtained by digitizing the test images [7], to evaluate the road feature extraction framework, at a 1:50,000 scale. This reference utilized as ground truth for an evaluation of the extracted roads. Also, the extracted roads are converted into the vector format using ArcGIS and spikes with a length of 150 meters (10 pixels) removed. The reference layer is a buffer layer with a width of $15 \mathrm{~m}$. The extracted roads fit within the reference roads network called as true positive (TP). Similarly, false positive (FP) and false negative (FN) calculated as in [39]. These Three metrics used to assess the accuracy of extracted road networks and calculated precision $(\mathrm{P})$, recall $(\mathrm{R})$, and $\mathrm{F} 1$-score [40]. Overlay of extracted roads of TP on the reference roads shown in Figure 14. Roads features extracted using RI from OLI images for the urban areas of Hyderabad, Chennai, and Bangalore with an overall F1-score of $85 \%$.

$$
F 1 \text { score }=2 \cdot \frac{\text { precision } \cdot \text { recall }}{\text { precision }+ \text { recall }}
$$

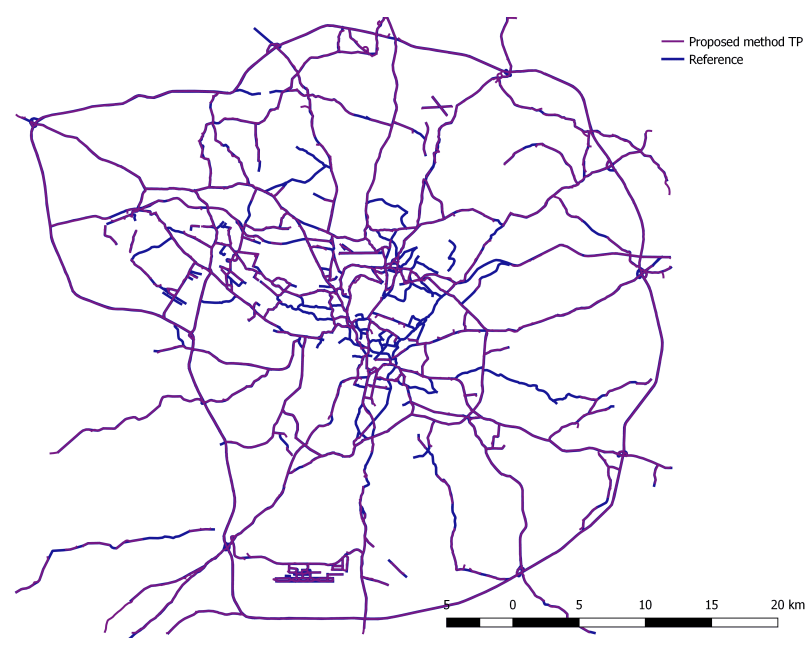

Figure 14. Overlay of vector layers, reference and proposed method (TP only) 


\subsubsection{Comparison}

Here, a classification algorithm i.e., pixel-based support vector machine (SVM) [41] classification with RBF kernel and polynomial order 2 is used to extract roads. Accuracy of the proposed methodology is comparing with the extraction of roads using the SVM on the same (B652) band combination. By using the SVM method, only major (width) roads get detected, due to limitation of SVM misclassification. The results of SVM and SATM images are shown in Figure 15. SVM method was able to classify the roads where roads are clear and remaining gaps are occurred due to the misclassification with building type. The accuracy of road feature extraction using the SVM and SATM methods given in Table 7.

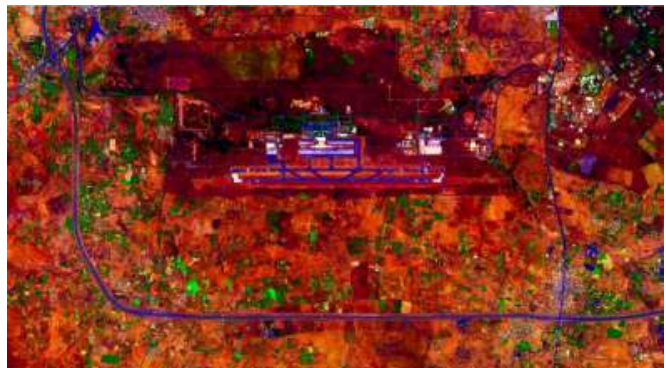

(a)

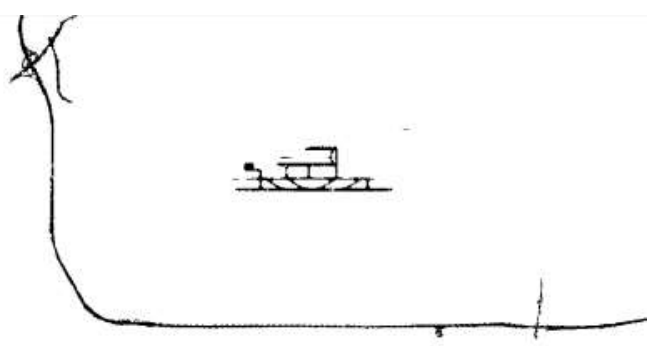

(c)

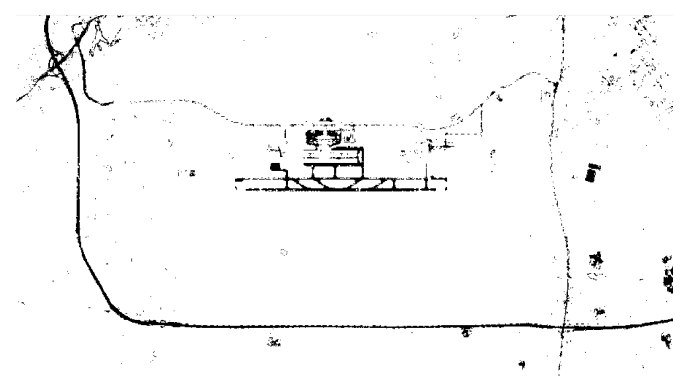

(b)

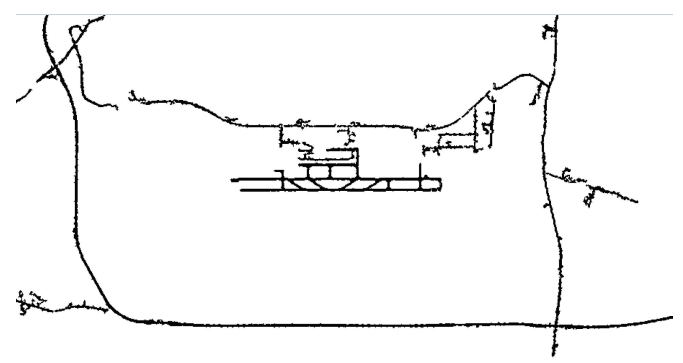

(d)

Figure 15. (a) Original (B652), (b) SVM, (c) After Post-processing of (b), (d) SATM

Table 7. Accuracy comparison

\begin{tabular}{cccccccc}
\hline & Extracted & TP & FP & FN & P & R & F1 \\
\hline SVM & 39 & 39 & 0 & 29 & 98.21 & 65.25 & 65.12 \\
SATM & 66 & 61 & 5 & 7 & 96.42 & 94.64 & 95.96 \\
\hline
\end{tabular}

\section{CONCLUSION}

In this paper, we proposed a methodology for road feature extraction based on road index using IAT and MRF (SATM and SMRF) from RS images. This methodology applied for the OLI images of the Indian urban areas. From the results, it observed that the extracted road widths are regularly greater than $30 \mathrm{~m}$ and airport runways with accurate. Also, observed that, in results, discontinuity is present in the road networks due to insufficient road width, presence of trees, and multi-storey buildings. These small gaps in between roads connected by the morphological operations, circle method that is, placing a circle at every endpoint of extracted roads and thinned. The remaining large gaps are not able to connect and consider for future work. The methodology brings advantages of extraction of the freeways and arterial roads by using OLI (LR images) instead of the HR images. Hence, the proposed methodology with RI is producing satisfactory results from OLI images. This methodology can extend for other sensors like Sentinel 2.

\section{ACKNOWLEDGMENT}

We wish to express our sincere gratitude to Dr. Shantanu Chowdhary, Director, National Remote Sensing Centre for their encouragement and guidance in bringing out this publication. 


\section{REFERENCES}

[1] J. Wang and Q. Zhang, "Applicability of a gradient profile algorithm for road network extractionsensor, resolution and background considerations," Canadian Journal of Remote Sensing, vol. 26, no. 5, pp. 428-439, 2000.

[2] S.Benjamin and L. Gaydos, "Spatial resolution requirements for automated cartographic road extraction," Photogrammetric Engineering and Remote Sensing, vol. 56, pp. 93-100, 1990.

[3] P. P. Singh and R. D. Garg, "Study of spectral reflectance characteristics of asphalt road surface using geomatics techniques," Int. Conf. on Advan. Comp., Commu. and Inform., pp. 516-520, 2013.

[4] M.Herald and D. A. Roberts, "Spectral characteristics of asphalt road aging and deterioration: implications for remote-sensing applications," Applied optics, vol. 44, no. 20, pp. 4327-4334, 2005.

[5] H. Xu, "Modification of normalised difference water index (ndwi) to enhance open water features in remotely sensed imagery," International Journal of Remote Sensing, vol. 27, no. 14, pp. 3025-3033, 2006.

[6] S. S. Bhatti and N. K. Tripathi, "Built-up area extraction using landsat 8 oli imagery," GIScience and Remote Sensing, vol. 51, no. 4, pp. 445-467, 2014.

[7] S. Das, T. T. Mirnalinee, and K. Varghese, "Use of salient features for the design of a multistage framework to extract roads from high-resolution multispectral satellite images," IEEE Transactions on Geoscience and Remote Sensing, vol. 49, no. 10, pp. 3906-3931, 2011.

[8] W. Wang, N. Yang, Y. Zhang, F. Wang, T. Cao, and P. Eklund, "A review of road extraction from remote sensing images," Journal of Traffic and Transportation Engineering (English Edition), vol. 3, no. 3, 2016.

[9] W. Shi, Z. Miao, and J. Debayle, "An integrated method for urban main-road centerline extraction from optical remotely sensed imagery," IEEE Transactions on Geoscience and Remote Sensing, vol. 52, no. 6, pp. 3359-3372, 2014.

[10] P. Gamba, F. Dell'Acqua, and G. Lisini, "Improving urban road extraction in high-resolution images exploiting directional filtering, perceptual grouping, and simple topological concepts," IEEE Geoscience and Remote Sensing Letters, vol. 3, no. 3, pp. 387-391, 2006.

[11] M. Fauvel, et al., "Spectral and spatial classification of hyperspectral data using svms and morphological profiles," IEEE International Geoscience and Remote Sensing Symposium, pp. 4834-4837, 2007.

[12] Z. Miao, et al., "An object-based method for road network extraction in vhr satellite images," IEEE J. of Selected Topics in Applied Earth Observations and Remote Sensing, vol. 8, no. 10, pp. 4853-4862, 2015.

[13] D. Chaudhuri, N. K. Kushwaha, and A. Samal, "Semi-automated road detection from high resolution satellite images by directional morphological enhancement and segmentation techniques," IEEE J. of Selected Topics in Applied Earth Observations and Remote Sensing, vol. 5, no. 5, pp. 1538-1544, 2012.

[14] M. O. Sghaier and R. Lepage, "Road extraction from very high resolution remote sensing optical images based on texture analysis and beamlet transform," IEEE Journal of Selected Topics in Applied Earth Observations and Remote Sensing, vol. 9, no. 5, pp. 1946-1958, 2016.

[15] Z. Miao, W. Shi, A. Samat, G. Lisini, and P. Gamba, "Information fusion for urban road extraction from vhr optical satellite images," IEEE Journal of Selected Topics in Applied Earth Observations and Remote Sensing, vol. 9, no. 5, pp. 1817-1829, 2016.

[16] T. Blaschke, et al, "Geographic object based image analysis-towards a new paradigm," ISPRS Journal of Photogrammetry and Remote Sensing, vol. 87, pp. 180-191, 2014.

[17] S. Dhingra and D. Kumar, "A review of remotely sensed satellite image classification," International Journal of Electrical and Computer Engineering, vol. 9, no. 3, pp. 1720-1731, 2019.

[18] N. Otsu, "A threshold selection method from gray-level histograms," IEEE Transactions on Systems, Man, and Cybernetics, vol. 9, no. 1, pp. 62-66, 1979.

[19] R. Adams and L. Bischof, "Seeded region growing," IEEE Transactions on Pattern Analysis and Machine Intelligence, vol. 16, no. 6, pp. 641-647, 1994.

[20] S. L. Horowitz and T. Pavlidis, "Picture segmentation by a tree traversal algorithm," J. ACM, vol. 23, no. 2, p. 368-388, 1976.

[21] R. K. Reddy, et al., "Comparative analysis of common edge detection algorithms using pre-processing technique," International Journal of Electrical and Computer Engineering (IJECE), vol. 7, no. 5, pp. 25742580, 2017.

[22] M. Basu, "Gaussian-based edge-detection methods-a survey," IEEE Transactions on Systems, Man, and Cybernetics, Part C (Applications and Reviews), vol. 32, no. 3, pp. 252-260, 2002.

[23] R. Taniguchi and E. Kawaguchi, "Road network extraction from landsat tm image," Third International 
Conference on Image Processing and its Applications, pp. 222-226, 1989.

[24] B. Liu, Z. Zhang, X. Liu, and W. Yu, "Edge extraction for polarimetric sar images using degenerate filter with weighted maximum likelihood estimation," IEEE Geoscience and Remote Sensing Letters, vol. 11, no. 12, pp. 2140-2144, 2014.

[25] F. Baselice, G. Ferraioli, and D. Reale, "Edge detection using real and imaginary decomposition of sar data," IEEE Transactions on Geoscience and Remote Sensing, vol. 52, no. 7, pp. 3833-3842, 2014.

[26] B. N. Saha and N. Ray, "Image thresholding by variational minimax optimization," Pattern Recognition, vol. 42, no. 5, pp. 843-856, 2009.

[27] S. Krishnamachari and R. Chellappa, "Multiresolution gauss-markov random field models for texture segmentation," IEEE Transactions on Image Processing, vol. 6, no. 2, pp. 251-267, 1997.

[28] N. Zhang and Q. Wu, "Effects of brovey transform and wavelet transform on the information capacity of spot-5 imagery," Proc. SPIE, 2007.

[29] A. Munsell, "A Grammar of Color: An Introduction to the Munsell Color System," The Strathmore Paper Company, 1921.

[30] S. Osher and L. I. Rudin, "Feature-oriented image enhancement using shock filters," SIAM Journal on Numerical Analysis, vol. 27, no. 4, pp. 919-940, 1990.

[31] L. Alvarez and L. Mazorra, "Signal and image restoration using shock filters and anisotropic diffusion," SIAM Journal on Numerical Analysis, vol. 31, pp. 590-605, 041994.

[32] Y. Zang, et al., "Joint enhancing filtering for road network ex-traction," IEEE Transactions on Geoscience and Remote Sensing, vol. 55, no. 3, pp. 1511-1525, 2017.

[33] J. Weickert, "Coherence-enhancing shock filters," Pattern Recognition, Springer, pp. 1-8, 2003.

[34] R. C. Dubes, et al., "Mrf model-based algorithms for image segmen- tation," Proce., 10th Int. Conference on Pattern Recognition, vol. 1, 1990, pp. 808-814, 1990.

[35] S. L. K. Reddy, et al., "Automatic road feature extraction using mrf from landsat-8 oli images," IEEE Recent Advances in Geoscience and Remote Sensing : Technologies, Standards and Applications (TENGARSS), pp. 15-20, 2019.

[36] C. Sujatha and D. Selvathi, "Connected component-based technique for automatic extraction of road centerline in high resolution satellite images," EURASIP Journal on Image and Video Processing, vol. 2015, no. 1, 2015.

[37] M. Yang, K. Kpalma, and J. Ronsin, ”A Survey of Shape Feature Extraction Techniques," 2008.

[38] S. L. K. Reddy, et al., "A novel method for water and water canal extraction from landsat-8 oli imagery," ISPRS - International Archives of the Photogrammetry, Remote Sensing and Spatial Information Sciences, vol. XLII-5, pp. 323-328, 2018.

[39] C. Heipke, H. Mayer, C. Wiedemann, and O. Jamet, "Evaluation of automatic road extraction," International Archives of Photogrammetry and Remote Sensing,pp. 47-56, 1997.

[40] D. R. Martin, C. C. Fowlkes, and J. Malik, "Learning to detect natural image boundaries using local brightness, color, and texture cues," IEEE Transactions on Pattern Analysis and Machine Intelligence, vol. 26, no. 5, pp. 530-549, 2004.

[41] D. Tuia, F. Pacifici, M. Kanevski, and W. J. Emery, "Classification of very high spatial resolution imagery using mathematical morphology and support vector machines," IEEE Transactions on Geoscience and Remote Sensing, vol. 47, no. 11, pp. 3866-3879, 2009.

\section{BIOGRAPHIES OF AUTHORS}

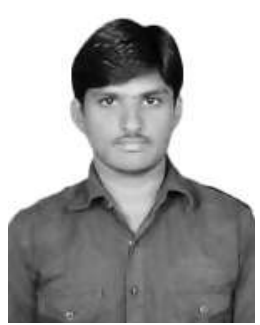

Sama Lenin Kumar Reddy received M.Tech. degree in Digital Communications from Kakatiya University (KU), India, in 2013, and working towards Ph.D. in Andhra University, Visakhapatnam, India. He is working as Senior Research Fellow (SRF) at National Remote Sensing Centre (NRSC), Indian Space Research Organization(ISRO), Hyderabad, India. His current areas of research interest are image processing, pattern recognition, visual perception and computational intelligence. 


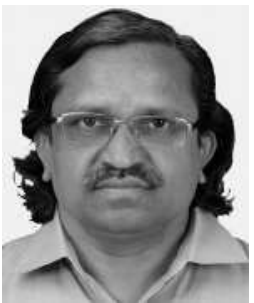

C. V. Rao is working as Scientist-SG, Technology and Innovation officer, NRSC, ISRO, Hyderabad, India. He received Ph.D. in image processing from JNTU Hyderabad in 2010 and also completed Three Ph.D. thesis under his guidance (currently guiding Three students) and several M.Tech students. He has published about 100 papers in international, national journals including conferences. His current areas of research interest are digital image processing, visual perception, computational intelligence and pattern recognition.

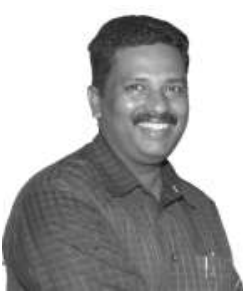

P.Rajesh Kumar received Ph.D. from Andhra University, Visakhapatnam, 2006. Currently, working as professor at Andhra University in the department of Electronics and communication Engineering. He has more than 20 years of teaching and 10 years of research experience. His current areas of research interest are image processing, signal processing and antenna theory.

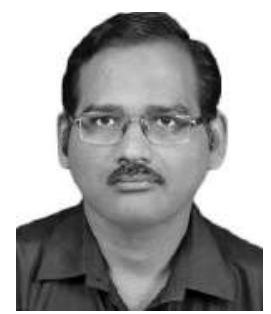

R.V.G.Anjaneyulu received M.Tech degree in Electronics and communication Engineering from Osmania University, Hyderabad, India, in 2000. Presently working as Head of the special products division, NRSC, ISRO, Hyderabad, India.

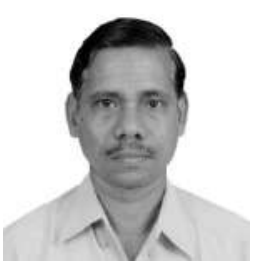

B.Gopala Krsihna received M.Tech degree from IIT Kharagpur. He is former Deputy Director of Data Processing, Products, Archival and Web Applications Area (DPPA \& WAA) at NRSC, Hyderabad, India. He has more than 170 publications to his credit in National and International journals. He has four software copyrights. His research interests include digital photogrammetry and mapping, geometrical data processing for remotely sensed data, planetary data processing, image mosaicking, stereo image analysis, and pattern matching. 